\title{
Experiment EPECUR for High Precision Measurements of the Pion Proton Cross Section in the Second Resonance Region
}

I.G. Alekseev, ${ }^{a}$ V.A. Andreev, ${ }^{b}$ I.G. Bordyuzhin, ${ }^{a}$ Ye.A. Filimonov, ${ }^{b}$ V.V. Golubev, ${ }^{b}$ A.B. Gridnev, ${ }^{b}$ D.V. Kalinkin ${ }^{*} a$ E.A. Konovalova, ${ }^{b}$ L.I. Koroleva, ${ }^{a}$ N.G. Kozlenko, ${ }^{b}$ V.S. Kozlov, ${ }^{b}$ A.G. Krivshich, ${ }^{b}$ V.A. Kuznetsov, ${ }^{b}$ B.V. Morozov, ${ }^{a}$ V.M. Nesterov, ${ }^{a}$ D.V. Novinsky, ${ }^{b}$ V.V. Ryltsov, ${ }^{a}$ M. Sadler, ${ }^{c}$ B.M. Shurygin, ${ }^{a}$ A.D. Sulimov, ${ }^{a}$ V.V. Sumachev, ${ }^{b}$ D.N. Svirida, ${ }^{a}$ V.I. Tarakanov ${ }^{b}$ and V.Yu. Trautman ${ }^{b}$

${ }^{a}$ Institute for Theoretical and Experimental Physics Moscow, Russia

${ }^{b}$ Petersburg Nuclear Physics Institute Gatchina, Leningrad district, Russia

${ }^{c}$ Abilene Christian University

Abilene, Texas, USA

In the search of light baryon resonances the main role is still held by partial wave analysis of the elastic pion-proton scattering. Yet most of the data available for PWA were obtained 20 years ago and earlier with an accuracy achievable at those times. One of the aims of EPECUR collaboration is to get qualitatively new experimental data in the pion-proton scattering. The experiment combines very high, better than $1 \mathrm{MeV}$, resolution in the invariant mass with high statistics. This makes it possible to look for narrow resonances with the coupling to the $\pi p$ channel as low as 5\%. One of such objects is 1-star $\mathrm{N}(1685)$ resonance. Over 3 billions triggers of both positive and negative pion beams in momentum range 820-1330 MeV/c were recorded since experiment start in 2009.

$X V$ International Conference on Hadron Spectroscopy

$4-8 / 11 / 2013$

Nara, Japan

\footnotetext{
${ }^{*}$ Speaker.

†E-mail: dmitry.kalinkin@gmail.com
} 


\section{Introduction}

EPECUR is an experiment devoted to a precise measurement of the differential cross section of the $\pi^{ \pm} p$ scattering and the total cross section of the $\pi^{-} p \rightarrow K^{0} \Lambda$ reaction in the region of incident pion momentum from 0.8 to $2 \mathrm{GeV} / \mathrm{c}$. Conducting "formation"-type experiment allows us to turn our high incident momentum resolution into a high invariant mass resolution of $\sim 0.6 \mathrm{GeV}$. Two different setups were designed: one for elastic reaction and one for inelastic, both being a non-magnetic spectrometers. Wire chamber utilization gives us minimal amount of matter on the particle paths what is good for angle measurement. The experiment also features high statistical precision - enough to define quantum numbers for a resonance. The measurement will contribute a new generation data for PWA along with measurement of the $\Lambda$-polarization in the reaction of $\pi^{-} p \rightarrow K^{0} \Lambda$ with an order of magnitude better precision than the data available before [四]. The data might also shed some light on the question of existence of the N(1685) cryptoexotic nonstrange member of the pentaquark antidecuplet predicted in [0] [3] and backed by numerous $\eta$ photoproduction experiments such as GRAAL[四].

\section{Elastic setup}

The elastic setup (see fig. 1) was built and commissioned at the universal beam channel 322 of the ITEP $10 \mathrm{GeV}$ proton synchrotron. Beam particles are registered using two sets of four $1 \mathrm{~mm}$ pitch wire proportional chambers (denoted as 1FCH1-4 and 2FCH1-4 on the figure) allowing to measure $\mathrm{X}$ and $\mathrm{Y}$ track projections. Particles are being scattered on the liquid hydrogen target

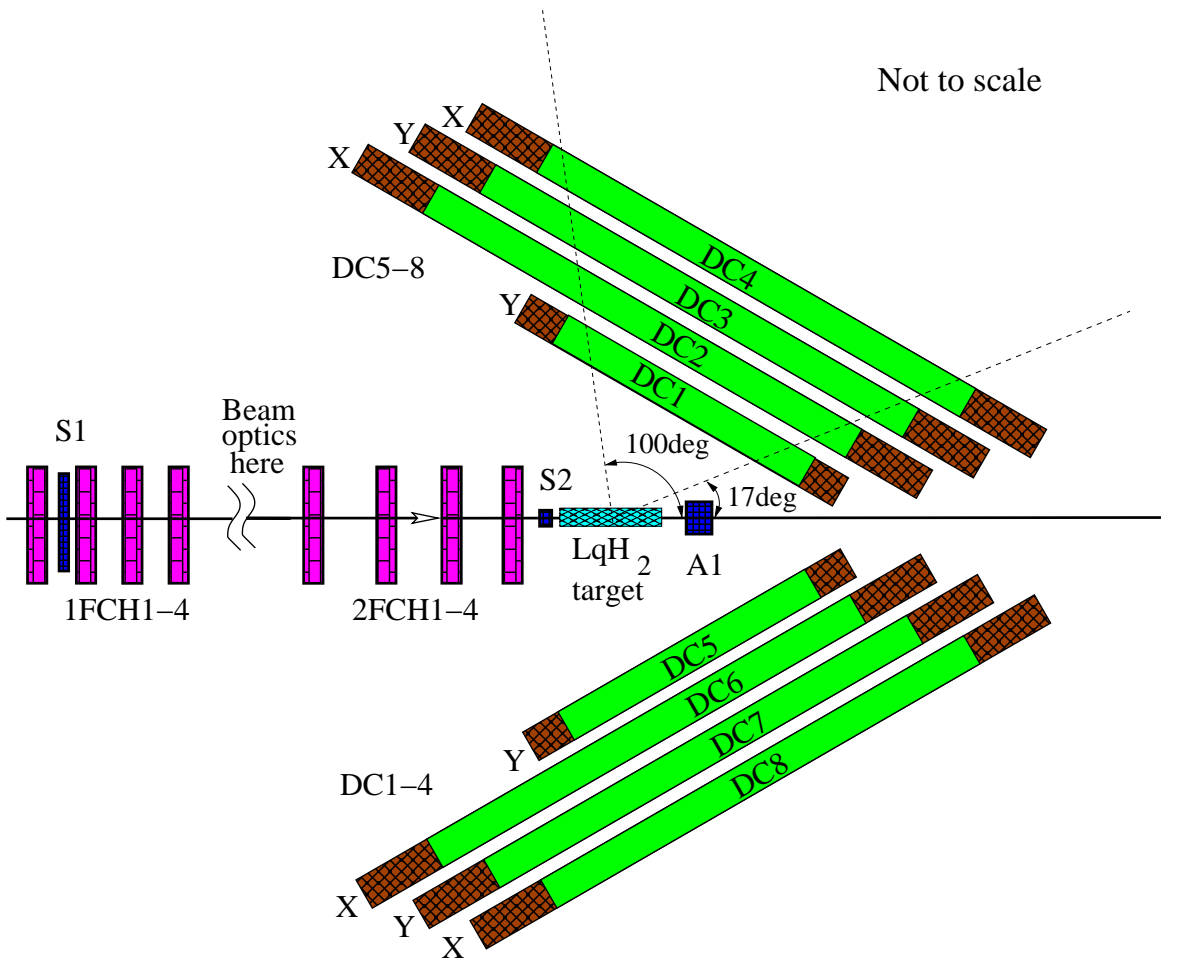

Figure 1: Schematic top view of the elastic setup. 
(denoted as $\mathrm{LqH}_{2}$ ) with beryllium outer shell and Mylar hydrogen container (40 $\mathrm{mm}$ in diameter and $250 \mathrm{~mm}$ along the beam). Secondary particles are registered using two arms of drift chambers (DC1-8) with hexagonal wire structure. Triggering is based on scintillation counters S1, S2, A1 and proportional chambers fast majoritary logic. Chamber readout and trigger electronics are based on FPGA's and microcontrollers[[]]. Magnetic field of the beam optics between the proportional chamber blocks is measured using NMR spectrometer.

\section{Elastic event selection}

Strong angular correlation imposed by kinematics of the elastic scattering can be used to select elastic events and do a particle identification. For that we take events having one particle in each of the two arms. Then we assume that the particle in the left arm is a pion, so we can predict the angle in which corresponding proton should have gone. Figure 2 shows how this prediction discrepancy is distributed with respect to the scattering angle. The vertical cluster corresponds to the hypothesis being right, the slanting cluster for it being wrong and the intersection area requires additional information for the resolution. In the case of the wrong hypothesis we take the event into a further processing with the swapped particle tags.
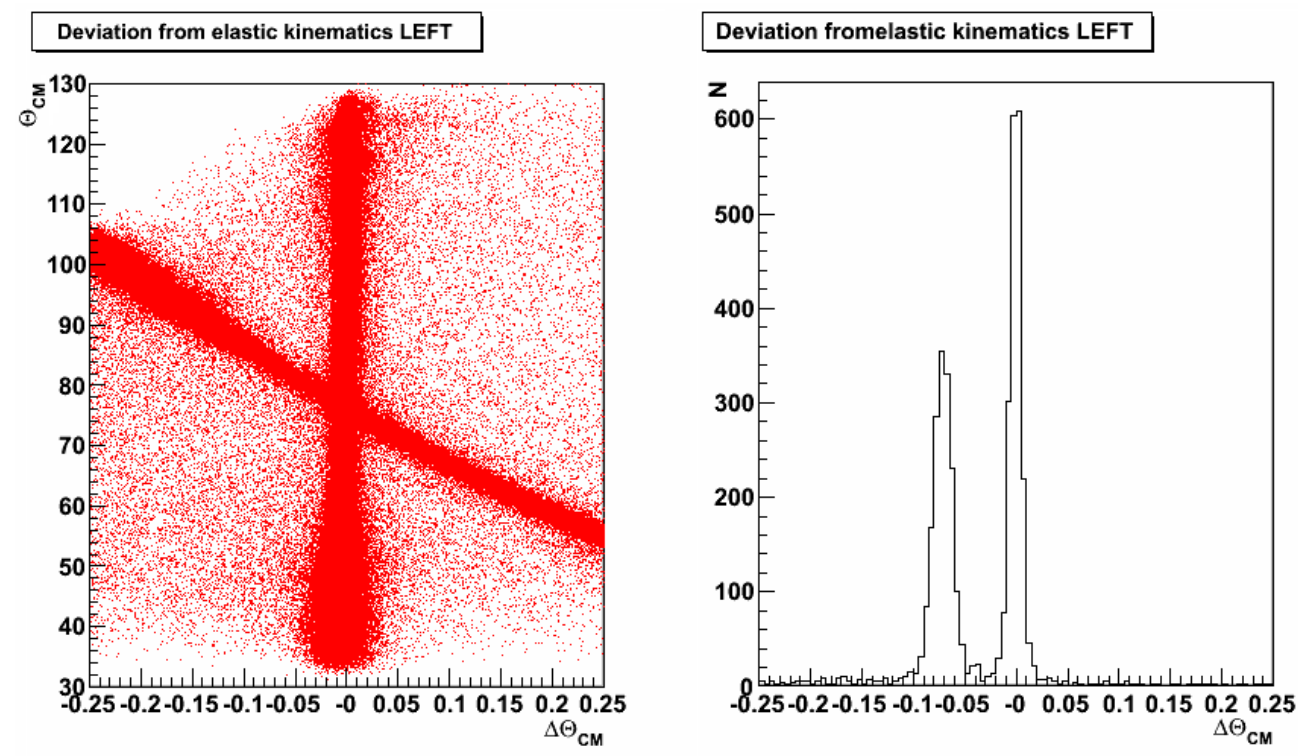

Figure 2: On the left: correlation between missprediction of the center of mass frame scattering angle $\Delta \Theta_{\mathrm{CM}}=\Theta_{\mathrm{CM}}-\Theta_{\mathrm{CM} \text { predicted }}$ versus measured scattering angle value $\Theta_{\mathrm{CM}}$. On the right: $\Theta_{\mathrm{CM}}=85^{\circ}$ slice of the left plot.

\section{Preliminary data}

Three billions of triggers were recorded since experiment start in 2009 [ 6 ]. Half of the available data were analyzed. Certain peculiarity around $P_{\text {beam }}=1020-1040 \mathrm{MeV} / \mathrm{c}$ is seen on our plots of differential cross section of the $\pi^{-} p$ reaction $[\square][[]$. Few sample plots of a cross section of the $\pi^{+} p$ elastic scattering are shown in figs. 3 and 4 . 

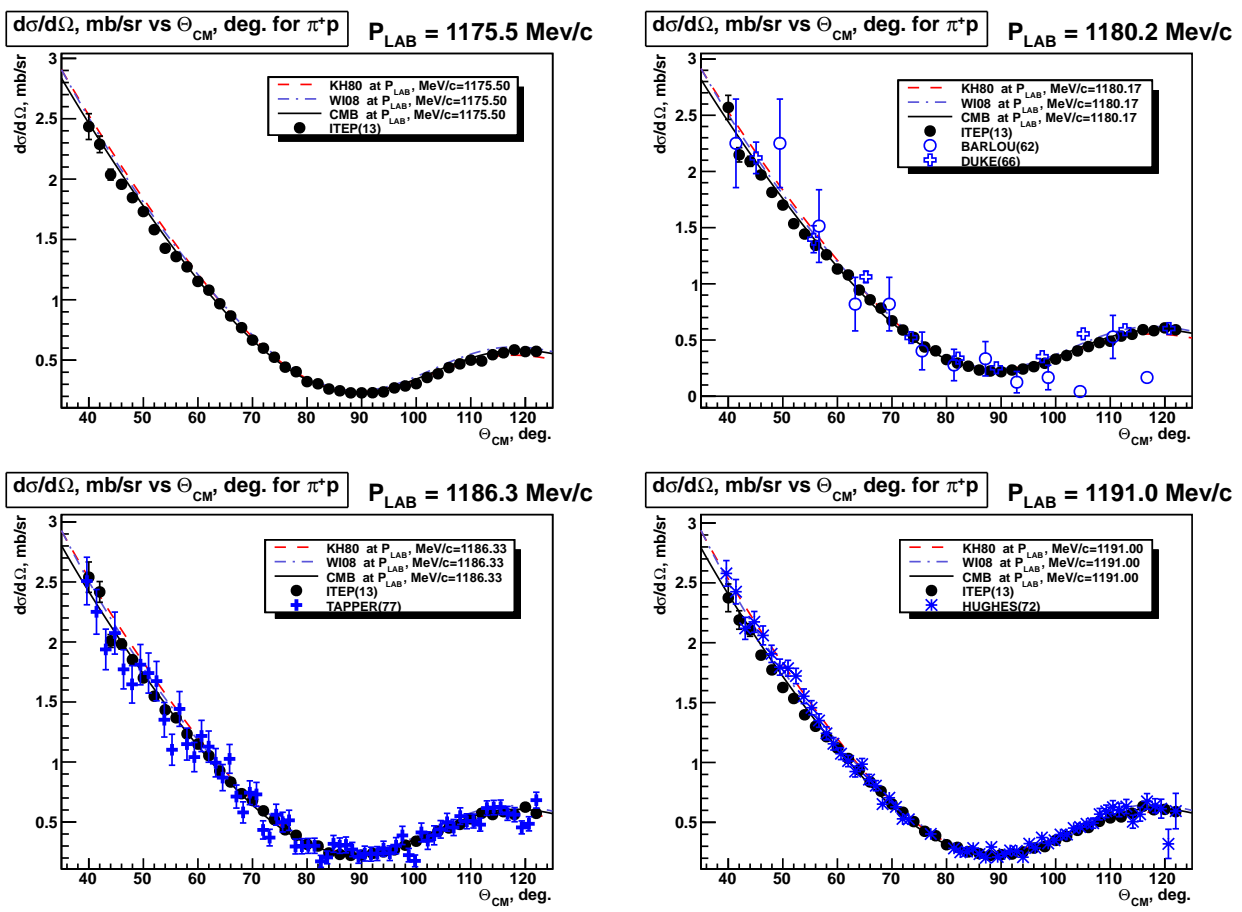

Figure 3: $\pi^{+} p$ elastic scattering differential cross section as a function of scattering angle for different pion beam momentum values. Black markers - our results (statistical errors only, preliminary), blue markers data from the other experiments.
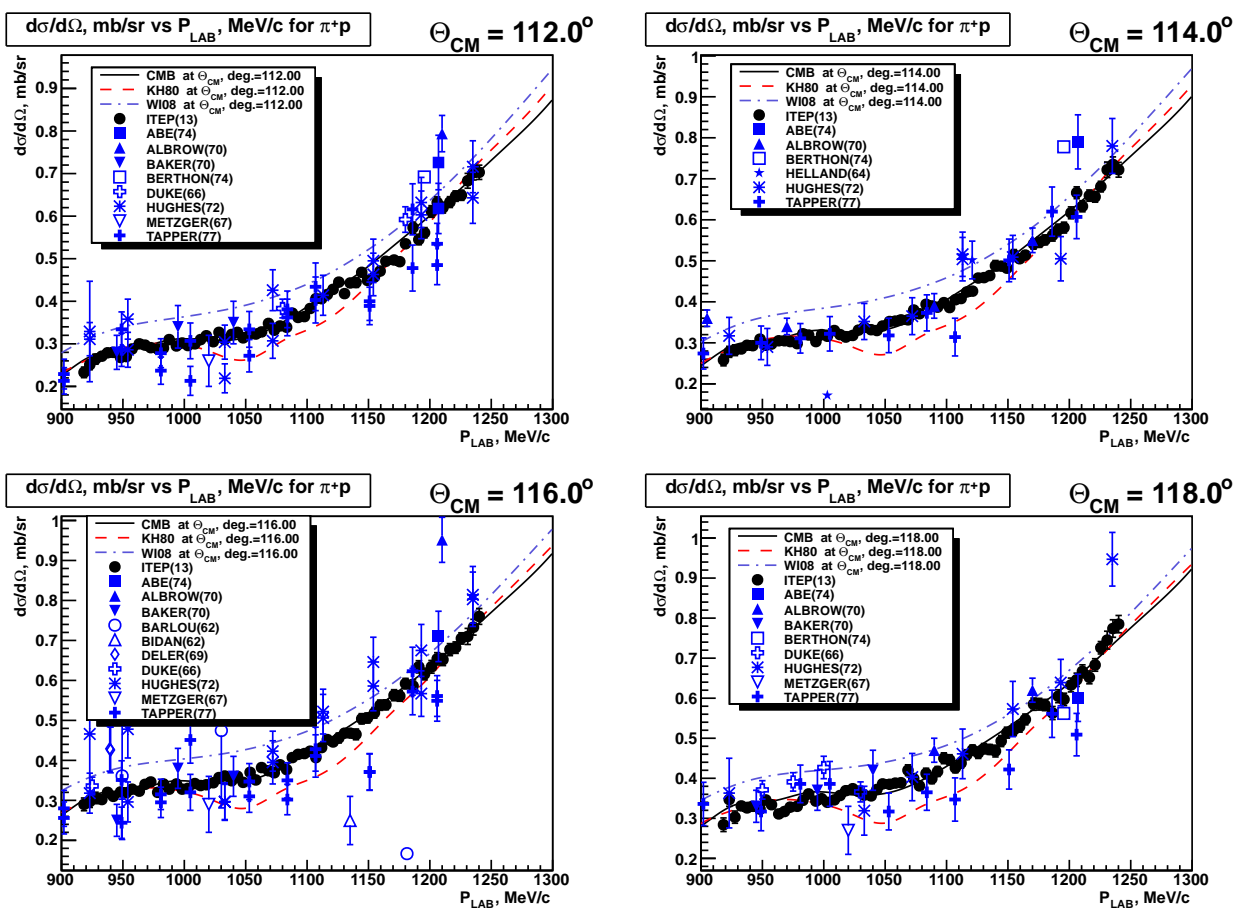

Figure 4: $\pi^{+} p$ elastic scattering differential cross section as a function of incident pion momentum for different scattering angle values. Black markers - our results (statistical errors only, preliminary), blue markers - data from the other experiments. 


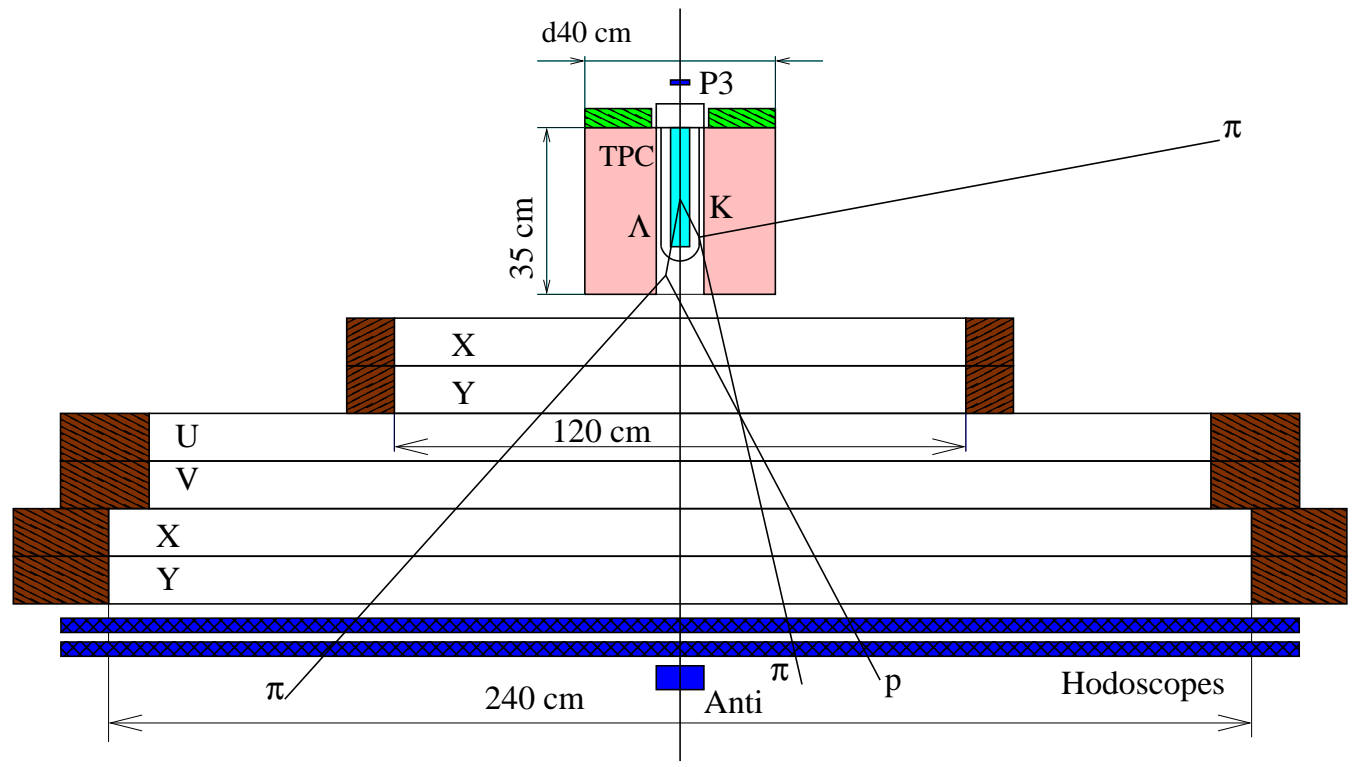

Figure 5: Schematic top view of the planned inelastic setup.

\section{Inelastic setup}

Our next step is to build an inelastic setup for the measurement of the $\Lambda K_{s}^{0}$-production events (fig. 5). The final state will be seen as a three pions and a proton. Most of the events will have all four particles going forward or three particles going forward and a pion going sideways.

The new setup will feature six drift chambers, four of which will be a newly produced large aperture ones, a plastic scintillator based TOF and a GEM TPC.

\section{Acknowledgements}

We thank ITEP accelerator team for providing an excellent beam. The experiment is supported by Russian Fund for Basic Research grants 09-02-00998a and 05-02-17005a.

\section{References}

[1] R.D. Baker et al., The Reaction $\pi^{-} p \rightarrow K^{0} \Lambda^{0}$ up to $1334 \mathrm{MeV/c}$, Nucl. Phys. B 141 (1978) 29-47.

[2] D. Diakonov, V. Petrov, M.V. Polyakov, Z. Phys. A 359, 305 (1997) [hep-ph/9703373].

[3] R. Arndt et al., Phys. Rev. C 69, 035208 (2004) huc1-th/0312126].

[4] V. Kuznetsov et al., Phys. Lett. B 647, 23 (2007) [hep-ex/080/.2316].

[5] I.G. Alekseev et al., Nucl. Instrum. Meth. A 578 (2007) 289-297 [physics/0701108].

[6] I.G. Alekseev et al., in proceedings of DSPIN'09 [hep-ex/0912.4681].

[7] I.G. Alekseev et al., in proceedings of NSTAR 2013 Workshop.

[8] A.B. Gridnev, in these proceedings, PoS (Hadron 2013) 099. 